SOCIAL RESEARCH REPORTS

ISSN: 2066-6861 (print), ISSN: 2067-5941 (electronic)

\title{
HEALTHY LIFESTYLE - HEALTHY MIND: THE ASSOCIATION BETWEEN HEALTH BEHAVIORS AND DEPRESSION AMONG ADULTS
}

\section{Dolev KAROLINSKY}

Social Research Reports, 2019, Vol. 11, Issue 1, pp. 138-149

The online version of this article can be found at: www. researchreports.ro

\section{https://doi.org/10.33788/srr11.1.10}

Published by:

Expert Projects Publishing House

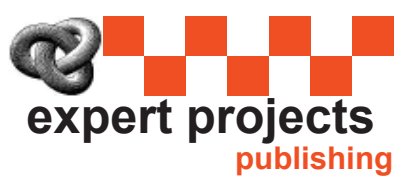

Covered by Index Copernicus International www.indexcopernicus.com

Directory of Open Access Journals www.doaj.org

On behalf of:

Center for Program and Social Development

Aditional services and information about Social Research Reports can be found at:

www.researchreports.ro 


\title{
HEALTHY LIFESTYLE - HEALTHY MIND: THE ASSOCIATION BETWEEN HEALTH BEHAVIORS AND DEPRESSION AMONG ADULTS
}

\author{
Dolev KAROLINSKY ${ }^{1,2}$
}

\section{Abstract}

Depressive disorders are one of the main causes of morbidity worldwide among adults. Although there are abundant scientific evidences on the relationship between sociodemographic and health characteristics and depression, theempirical evidence addressing the impact of health behaviors on depressive symptoms remained inconclusive. The current review describes in detail the associations between several main healthy lifestyle and depression among adults, including the possible influence of the locus of control component on compliance with health behaviors. In addition, this article discusses on the meaning of a "healthy lifestyle" as a predictor for depression, informed by methodological and conceptual challenges of biosocial approaches.

Keywords: depressive symptoms, healthy lifestyle, health behaviors, locus of control, biosocial approach, synergistic effect.

\footnotetext{
${ }^{1}$ The Max Stern Yezreel Valley College, Yezreel Valley, ISRAEL. E-mail: dolevka@ gmail.com

${ }^{2}$ West University Timisoara, Timisoara. ROMANIA.
} 


\section{Introduction}

Depressive disorders, characterized by low mood, a reduced ability to experience pleasure (anhedonia) and a combination of cognitive symptoms, such as low self-esteem, and physiological disturbances, such as insomnia or loss of appetite, are one of the most debilitating mental illnesses in the modern world that significantly impairs social and occupational functioning (Sibille \& French, 2013; Smithson \& Pignone, 2017). One of the findings of mental health surveys conducted by the WHO (World Health Organization) since 2001 and included Israel, was that the point prevalence rate of major depressive disorder (in the last 12 months) is approximately $6 \%$ during the last decade (Kessler et al., 2010; Kessler \& Bromet, 2013). During the years 2003-2004 the WHO survey was conducted in Israel and similar rate of major depressive disorder was reported (5.9\%) (Levinson et al., 2007).

Regardless of the level of severity, depressive symptoms, whether major, moderate, or sub-threshold were related to increased risk for comorbidity, disability, decreased quality of life and mortality (Geulayov et al., 2007). The evidence of association between chronic conditions and depression is abundant (Caneo et al., 2016). In an international study a direct relationship was found between the number of depression symptoms, and self-reported poor health status and functioning (Herrman et al., 2002). There are correlation between depression and various chronic conditions, such as cancer, asthma, obesity, heart coronary diseases and diabetes (Chapman, Perry \& Strine, 2005; Katon, 2004; Kessler \& Bromet, 2013). However, chronic conditions might be independent risk factors for depression; several cohort studies demonstrated casual relationships in the association between different chronic conditions and depression. For instance, one study showed that each of the following conditions increased the risk for depression occurrence: cardiovascular diseases, metabolic syndrome, autoimmune diseases and migraine. Moreover, several studies demonstrated dose-response between the number of physical illnesses and depression severity (Caneo et al., 2016).

Empirical studies have consistently demonstrated associations between socioeconomic and demographic variables and the prevalence of depressive disorders both in Israel and worldwide (Iancu et al., 2003; Kaplan et al., 2010; Kessler \& Bromet, 2013; Piccinelli \& Wilkinson, 2000). Yet, those studies cannot capture the full picture in its entirety because of overlooking other important factors that may be related to the occurrence of depression, such as healthy behaviors and lifestyle (Elizur et al., 2010). Israel has not been an exception, as most of the studies conducted in this country did not focus on the independent influence of health patterns on depression, with emphasis on health-related behaviors (Iancu et.al, 2003; Kaplan et.al., 2010). The purpose of the current review is to describe in detail the associations between healthy lifestyle and depression among adults, with emphasis on the following behaviors: vegetables and fruits consumption, physical activity, weight watching and smoking status. 


\section{Methodology}

\section{Search strategy and selection criteria}

The sources for articles searching were "Pubmed" and "Google scholar", using the following search terms: 'Depression', 'Depression and Health', 'Mental health', 'Depression and Health behaviors' and 'Depression and Healthy Lifestyle'. The search was limited to English only. Studies were included in case that theyfocus on health behaviors and depression among the adult population. In addition, articles that discuss the benefits or risks of health behaviors and related socio-cultural issues were also included.

\section{Results}

According to WHO recent report, negative health behaviors, such as physical inactivity, poor nutrition, and smoking are among risk factors for morbidity and mortality in the world (WHO, 2009). In particular, the unhealthy lifestyle was found as either directly or implicitly related to development of mental disorders, including depression. Moreover, the co-existence of several negative health behaviors may exacerbate the influence (Cabello et al., 2017). For instance, Adjibade et al. (2018) found that unhealthy diet, smoking status, and obesity were independently associated with the risk of developing depressive symptoms; in addition, the findings show that increased number of healthy lifestyle behaviors was associated with decreased risk of depressive symptoms; finally, it was found that $14 \%$ of cases were attributed to the combination of unhealthy diet, overweight (BMI) and smoking. In essence, the scholars sought to bridge the empirical lacunae and study the combined effect of those variables by calculating a Healthy Lifestyle Index (HLI) which included several health behaviors, with the purpose of further investigating its association with the incidence of depressive symptoms (Adjibade et al., 2018). Another research that was conducted by Japanese scholars revealed four variables that were significantly associated with the prevalence of depressive symptoms: 1) insufficient sleep, 2) a poorly-balanced diet, 3) snacking between meals and 4) physical inactiveness (Furihata et al., 2018).

A literature review revealed that four of the main indicators of health behaviors predicting the depression occurrence are level of vegetables and fruits consumption, physical activity level, weight watching status and smoking status, as well as their combinations. The following sections present a detailed description of these variables. 


\section{Vegetables and fruits consumption}

Consumption of vegetables and fruits supplies essential vitamins and minerals to the human body and constitutes a source of phytochemicals, serving as antioxidants and anti-inflammatory agents contributing to the body's defense mechanisms (Slavin \& Lloyd, 2012). The National Institute for Cancer in the USA recommends on consumption of 5 doses of vegetables and fruit every day. The decision on this dose relies on the recommendation of the WHO, according to which the recommended daily amount of vegetables and fruits is 400 grams (not including potatoes) (WHO, 2003). In a meta-analytic study which included more than 200,000 people, the highest levels of consumption of vegetables and fruits (by comparison to the lowest consumption levels of both vegetables and fruits) were found to reduce the risk for depression by $14 \%$ and $11 \%$, respectively (Liu et al., 2016). Scientific evidences show a relationship between a lack of antioxidants and psychiatric disorders. Vegetables and fruits are considered to be enriched foods by fiber, vitamins, minerals, antioxidants, flavonoids and phytochemicals. These nutrients are a protective factor against depression. In the biological system, some of the agents which are found in vegetables and fruits, such as beta-carotene, vitamin E and vitamin C are efficient antioxidants (Liu et al., 2016).

Particular dietary deficiencies may explain the association between vegetables and fruits consumption and depression. For instance, a lack of folates involved in the production of neurotransmitters or vitamin B12 may lead to an increase in homocysteine levels. A high level of homocysteine may lead to processes that will cause brain damage and thus increase the risk for depression. In addition, vitamin B6 is part of the production process of neurotransmitters (like serotonin) that contributes to mood enhancement. Therefore, a deficiency of this vitamin may also be related to higher risk for depression (Saghafian et al., 2018). Magnesium deficiency which is associated with inflammatory processes can in turn increase the risk for depression, and deficiency in zinc leads to increased neurological, physical, and psychopathological symptoms which are associated with a depressive disorder (Liu et al., 2016).

However, with regard to the relationship between vegetables and fruits the findings are still inconsistent. Several studies found that while fruits intake was significantly related to decreased risk for depression, vegetables intake was not found to be significant in reducing the risk for depression, and vice versa. Other studies did not reveal any association between vegetables and fruits consumption and depression (Saghafian et al., 2018). Moreover, a few studies even indicated harmful influence of vegetables and fruit consumption on the development of depression, i.e. higher consumption levels were related to higher risk for depression (Liu et al., 2016).

\section{Physical activity}

The current recommendations for physical activity performance were published by the Health Department of the United States in the year 2008 (Buchner et al., 2008). These recommendations were adopted by the WHO (WHO, 2010). According to the updated recommendations one should perform aerobic physical activity 
that accumulates either to 150 minutes per week of moderate-intensity (activity involving a moderate rise in the breathing rate and in heartbeats, a light feeling of heat and sweat) or 75 minutes per week of vigorous-intensity (an activity involving a large rise in the breathing rate and heartbeats, an intense feeling of heat and sweat) or an equivalent combination of both intensities (Buchner et al., 2008).

Research evidences show that physical activity has a contribution to physical health status (Galily et al., 2012). Performing physical activity on a regular basis contributes to prevention of a variety of diseases and chronic conditions, such as cardiovascular diseases, diabetes, cancer diseases, hypertension, obesity, etc. (Warburton, Nicol, \& Bredin, 2006).

Physical activity has consistently emerged as an important predictor of depressive symptoms and depression. Epidemiological studies indicated that doing exercise at the recommended level was associated with few depressive symptoms, while non-exercising or exercise at an inadequate level was related to an enhanced probability for developing depressive symptoms (Mammen \& Faulkner, 2013; Teychenne, Ball \& Salmon, 2008). The majority of studies demonstrated a positive relationship between engagement in physical activity and mental health (Mammen \& Faulkner, 2013; Teychenne et al., 2008). It was found that compliance with the recommended level of physical activity (at least 150 minutes per week) reduced the risk for developing depression in 19\%-27\% (Mammen \& Faulkner, 2013). An additional study identified the minimal level of physical activity that may serve as a protective measure for depression occurrence. It was found that daily physical activity of 10-29 minutes reduces the risk for depression by $10 \%$, and that there is a dose-response relationship between the average daily duration of physical activity and the risk for developing depression; a longer duration of activity was related to a greater reduction of the risk (Lucas et al., 2011).

Studies revealed that physical exercises increased the release of serotonin thereby acting as an "anti-depressant". In addition, long-terms effects of routine physical activity may enhance neuroendocrine regulation axis thereby leading to normalization of cortisol levels, alongside with increased release of endorphins that contribute to mood improvements (Sarris, Kavanagh \& Newton, 2008). In addition to biological benefits, exercise affects the moderators of self-efficacy and self-esteem and thus may help to reduce the development of depression. Moreover, exercise-oriented sessions are an opportunity to enlarge supportive social network, thereby diminishing the risk for developing depressive symptoms (Deslandes et al., 2009; Sarris et al., 2008).

However, several studies found that physical activity was not related to depression, while others found that engagement in physical activity serves as a protective factor for depression among women, but not among men (Mammen\& Faulkner, 2013). Furthermore, it has been found that for men, the desire for muscularity is a component of their body image and that a higher drive for muscularity correlates with a lower level of well-being, indicating negative relationship between exercise and depressive symptoms (Swami et al., 2018). 


\section{Weight watching}

Obesity is an independent risk factor for metabolic disorders. Moreover, it might result in serious health consequences for individuals. Scientific evidences showed that there are around 20 co-morbidities which are related to overweight and obesity. In addition, obese individuals more frequently suffer from significant joint pains disorders than non-obese people. Moreover, obesity may result in social and psychological impairments (Djalalinia, et al., 2015).

A longitudinal meta-analysis showed bidirectional associations between depression and obesity: obese persons had a 55\% increased risk of developing depression over time, whereas depressed persons had a $58 \%$ increased risk of becoming obese (Luppino et al., 2010). It is noteworthy that the effect of obesity on developing depressive symptoms was stronger in American studies, indicating the difference between sociocultural mechanisms which could be more stringent in one culture that in the other (Atlantis \& Ball, 2008).

Weight issues were found to affect developing depressive symptoms both biologically and psychologically. Biologically, obesity causes inflammatory conditions in the body, which are known to be associated with depression. In addition, obesity can affect the hypothalamus-pituitary-adrenal glands (HPA axis). It is known that regulation problems in this axis are associated with depression (Luppino et al., 2010).

The social aspects, such as perceptions of ideal body by Western culture, as well as media influence, increase the possibility of experiencing distress because of physical and therefore social inadequacy. The feeling of inadequacy, accompanied by dissatisfaction with their bodies, and negative effect on the self-image, in turn, may lead to developing depressive symptoms (Atlantis \& Ball, 2008; Derenne \& Beresin, 2006; Hoek et al., 2005).

\section{Smoking}

Consumption of tobacco is expected to be one of the prominent health problems all over the world in terms of mortality and disability (Klungsoyr et al., 2006). Previous studies found that in developed countries, smoking constitutes a primary risk factor for early death, with $28 \%$ of the cases attributed to lung cancer. More than 400,000 cases of death and $30 \%$ of all cancer diseases were attributed to smoking in the USA (Bergen \& Caporaso, 1999).

There is a bidirectional relationship between depression and smoking, as depression is more prevalent among smokers, and, similarly, smoking is more prevalent among depressed individuals than in non-depressed ones (Freedland, Carney, \& Skala, 2005). It seems that there is a dose-response relationship between smoking levels and depression. In a study done in Norway it was found that higher smoking amounts (as based on the number of cigarettes and years of smoking) was associated with higher risk for developing major depression (Klungsoyr et al., 2006). Prolonged smoking period is also associated with subclinical depression (Weinberger et al., 2012). The negative effect of smoking is associated with increased depressive symptoms and with decreased motivation to comply with treatment for depression (Sarris et al., 2014). 
However, empirical evidence regarding the possibility of a causal association between smoking and depression remains inconsistent. For instance, findings from two researches have shown that the association between smoking and the occurrence of depression ismerely circumstantial. They concluded that this association was derived from genetic factors, which increased both the risk for smoking and the vulnerability to depression (Freedland et al., 2005). In addition, smoking as marker of unhealthy behavior is embedded in the framework of social acceptance, as in the US, where it varied tremendously from time to time, thereby affecting people's likelihood of smoking, as well as the degree to which genetic susceptibility to it is related with smoking behavior (Boardman, Blalock, \& Pampel, 2010).

\section{Adherence to healthy lifestyle: the locus of control component}

Culturaland social characteristics may play a role in health perceptions, compliance with health behaviors etc. For instance, one of these domains is the Health Locus of Control (Al-Krenawi, 2005; Baron-Epel et al., 2005; Cohen \& Azaiza, 2007). The locus of control expresses the perception of control that people have or do not have over their life events, such as their health status. The focus of health control may be internal (i.e., people believe that their health status is influence by their behaviors and mode of functioning) or external (i.e., the people believe that their health depends on force majeure or on fate) (Cohen \& Azaiza, 2007). Studies show that people with an internal locus of health, unlike those with an external one, tend to proactively follow a healthy lifestyle and take action to improve their health. For instance, it was shown in previous studies that the external locus of health was negatively related to high rates of physical activity, while internal control locus of health was positively associated with high rates of exercise (Cohen \& Azaiza, 2007). In addition, individuals who have an external locus of control of health tend to suffer from depression and having lower compliance with treatment for depression because of their basic belief that they can not affect their health status, including their mental health condition (Shojaee, 2014).

\section{Discussion}

The review of the research from the last decades shows empirical lacunae in regard to those predictors of depression in adults which are related to health behaviors. There is a consistent support for the negative impact of unhealthy behaviors upon mental health in general and developing depressive symptoms, ranging from subclinical to major depression (Adjibade et al., 2018). However, there are two major flaws in the current research body: (1) health behaviors have been mostly studied independently and in separation; therefore, their cumulative and joint influence still needs further investigations; (2) gaps and ambiguities in findings, such as absence of direct causality between smoking and depression or unexpected gender differences regarding physical exercise. These problems highlight the challenges of the biosocial approaches in establishing links between social environments and healthy behaviors, both methodologically and concep- 
tually (Short \& Mollborn, 2015). The whole is larger than the sum of its parts. Health-related behavioral patterns do not occur in isolation; rather, they cluster together and are cumulative in specific population subgroups, thereby calling for a more holistic approach to health. Furthermore, since clustering of unhealthy behaviors have synergistic effect, their combination is even more detrimental to health that any added individual effects of health behaviors (Stringhini et al., 2010; Kaczynski et al., 2008).

Further exploring the construct of "healthy lifestyle" should comprise a dimension of Locus of Control (Cohen \& Azaiza, 2007). Negative health behaviors and tendency to depression may be affected independently by the pattern of control locus of heath; individuals who have an external locus of control of health tend to suffer from depression and having lower compliance with treatment for depression because of their basic belief that they cannot affect their health status, or their mental health (Shojaee, 2014). Therefore, locus of health control may constitute one of the indicators for "healthy lifestyle" of the individuals by directly influencing their health choices. Integrating the dimension of locus of control into the construct of "lifestyle" is informed by the research that identified links between cognitive processes such as decision-making and framing and health behavior (Reyna et al., 2015). In this context, implementing policies that emphasize the norms of healthy eating may be more effective than those which regulate food prices (Zhang et al., 2014).

\section{Conclusion}

Health behaviors identified as major predictors for depression in adults should be further crystallized into a synergistic construct of what can be deemed "healthy lifestyle". Such a construct is informed by the premises of biosocial approaches that link between social and biological influences (Adjibade et al., 2018). More empirical insights are needed into the nature of associations between the variables of health behaviors and other moderators, such as locus of control or cultural perceptions. Exploring internal associations between variables, such as physical activity and obesity, may lead to developing a valid index of a healthy lifestyle. The main purpose of refining the clusters is to create a conceptual and methodological framework of reference which addresses biosocial processes in the interdisciplinary space.

Identifying and exploring the clusters of health-related behaviors in a population can contribute to better planning of prevention and designing intervention strategies (Schuit, van Loon, \& Ocke, 2002). Such planning should address sociocultural and demographic variance between target populations and consider existing norms and values, codes of behavior, attitudes and perceptions of "being ill" or "healthy" in the given society. People with similar social locations are found to exhibit similar health behavioral patterns, even though those behaviors might change with age ( $\varnothing$ vrum, Gustavsen, \& Rickertsen, 2014). Future interventions should be based on understanding of the dynamic interplay between and within various environments and biological processes; for example intervention related 
to obesity should include inputs such as poverty and local food environment (Ip et al., 2013).

As health behaviors affect and shape health and well-being of individuals and populations, and in particular their mental health, there is a need to expand the scholarly approach by shifting the perspective and viewing health behaviors as multidimensional constructs, related to individual and society health lifestyles; those behaviors are affected and penetrated by ideologies, policies, and institutions; therefore, they can be understood by exploring the triangle of social, cultural, and biological aspects. Consequently, the future research will situate the individual and public health in context of multiple influences and advance the methodological and conceptual representations of this complexity.

\section{References}

Adjibade, M, Lemgne, C., Chantal, J. Hercberg, s. Galan, P., Assmann K.E, \&Kesse-Guyot, E. (2018). Prospective association between combined healthy lifestyles and risk of depressive symptoms in the French NutriNet-Sante Cohort, Journal of Affective Disorders, 238(1), 554-562.

Al-Krenawi, A. (2005). Socio-political aspects of mental health practice with Arabs in the Israeli context. The Israel Journal of Psychiatry and Related Sciences, 42, 126-136.

Atlantis, E. \& Ball, K. (2008). Association between weight perception and psychological distress, International Journal of Obesity, 32(4), 715-721.

Baron-Epel, O., Kaplan, G., Haviv-Messika, A., Tarabeia, J., Green, MS. \& Kaluski, DN. (2005). Self-reported health as a cultural health determinant in Arab and Jewish Israelis MABAT--National Health and Nutrition Survey 1999-2001. Social Science and Medicine, 61, 1256-1266.

Bergen, A.W. \& Caporaso, N. (1999). Cigarette smoking. Journal of the National Cancer Institue, 91, 1365-1375.

Boardman, J.D., Blalock, C.L. \& Pampel, F.C. (2010) Trends in the genetic influences on smoking. Journal of Health and Social Behavior, 51, 108-123.

Buchner, D., \& Association NAT. (2008). 2008 Physical Activity Guidelines for Americans. Health Learning.

Cabello, M., Miret, M., Caballero, F.F., Chatterji, S., Naidoo, N., Kowal, P., ... Ayuso-Mateos, J.L. (2017). The role of unhealthy lifestyles in the incidence and persistence of depression: a longitudinal general population study in four emerging countries. Globalization and Health. 13. doi: 10.1186/s12992-017-0237-5.

Caneo, C., Marston, L., Bellon, J.A. \& King, M. (2016). Examining the relationship between physical illness and depression: Is there a difference between inflammatory and non inflammatory diseases? A cohort study. General Hospital Psychiatry, 43, 71-77. doi: 10.1016/j.genhosppsych.2016.09.007.

Chapman, D.P., Perry, G.S. \& Strine, T.W. (2005). The vital link between chronic disease and depressive disorders. Preventing Chronic Disease, 2, 1-10.

Cohen, M., \& Azaiza, F. (2007). Health-promoting behaviors and health locus of control from a multicultural perspective. Ethnicity and Disease, 17(4), 636-642.

Derenne, J.L. \& Beresin, E.V. (2006). Body image, media, and eating disorders. Academic Psychiatry, 30, 257-261.

Deslandes, A., Moraes, H., Ferreira, C., Veiga, H., Silveira, H., Mouta, R., ... Laks, J. 
(2009). Exercise and mental health: many reasons to move. Neuropsychobiology, 59, 191-198.

Djalalinia, S., Qorbani, M., Peykari, N., \& Kelishadi, R. (2015). Health impacts of Obesity. Pakistan Journal of Medical Sciences, 31, 239-42.

Elizur, E.S., Tyano, S., Munitz, H., \& Neumann, M. (2010). Selected chapter in psychiatry. Tel Aviv, IL: PROBOOK.

Freedland, K.E., Carney, R.M., \& Skala, J.A. (2005). Depression and smoking in coronary heart disease. Psychosomatic Medicine, 67(Suppl 1), S42-46.

Furihata, R., Konno, C., Suzuki, M., Takanashi, S., Kaneita, Y. Ohida T, \& Uchiyama, M. (2018). Unhealthy lifestyle factors and depressive symptoms: a Japanese general adult population survey, Journal of Affective Disorders, 234, 156-161.

Galily, Y., Tamir, I., Meckel, Y. \& Eliakim, A. (2012). Socio-cultural characteristics of physical activity habits in Israel (1992-2008). International Journal of Sociology and Social Policy, 32, 461-479.

Geulayov, G., Lipsitz, J., Sabar, R. \& Gross R. (2007). Depression in primary care in Israel. The Israel Medical Association journal, 9, 571-578.

Herrman, H., Patrick D.L., Diehr, P., Martin M.L., Fleck M., Simon G.E., \& Buesching D.P. (2002). Longitudinal investigation of depression outcomes in primary care in six countries: the LIDO study. Functional status, health service use and treatment of people with depressive symptoms. Psychological Medicine, 32, 889-902.

Hoek, H.W., van Harten, P.N., Hermans, K.M., Katzman, M.A., Matroos, G.E., \& Susser, E.S. (2005). The incidence of anorexia nervosa on Curacao. The American Journal of Psychiatry, 162, 748-752.

Iancu, I., Horesh, N., Lepkifker, E., \& Drory, Y. (2003). An epidemiological study of depressive symptomatology among Israeli adults: prevalence of depressive symptoms and demographic risk factors. Israel Journal of Psychiatry Related Science, $40,82-89$.

Ip, E.H., Rahmandad, H., Shoham, D.A., Hammond, R., Huang, T.T., Wang, Y., \& Mabry, P.L. (2013). Reconciling statistical and systems science approaches to public health. Health Education and Behavior, 40(1Suppl), 123S-131S.

Kaczynski, A., Manske, S., Mannell, R., \& Grewal, K. (2008). Smoking and physical activity: a systematic review. American Journal of Health Behavior, 32, 93-110.

Kaplan, G., Glasser, S., Murad, H., Atamna, A., Alpert, G., Goldbourt, U., \& Kalter-Leibovici, O. (2010). Depression among Arabs and Jews in Israel: a population-based study. Social Psychiatry and Psychiatric Epidemiology, 45, 931-939.

Katon, W.J. (2004). The many faces of depression in primary care. Journal of General Internal Medicine, 19, 893-895.

Kessler, R.C., \& Bromet, E.J. (2013). The epidemiology of depression across cultures. Annual Review of Public Health, 34, 119-138.

Kessler, R.C., Birnbaum, H.G., Shahly, V., Bromet, E., Hwang, I., McLaughlin, K.A., ... Stein, D.J. (2010). Age differences in the prevalence and co-morbidity of DSM-IV major depressive episodes: results from the WHO World Mental Health Survey Initiative. Depression and Anxiety, 27, 351-364.

Klungsoyr, O., Nygard, J.F., Sorensen, T., \& Sandanger, I. (2006). Cigarette smoking and incidence of first depressive episode: an 11-year, population-based follow-up study. American Journal of Epidemiology, 5, 421-432.

Levinson, D., Zilber, N., Lerner, Y., Grinshpoon, A., \& Levav, I. (2007). Prevalence of 
Mood and Anxiety Disorders in the Community: Results from the Israel National Health Survey. The Israel Journal of Psychiatry and Related Sciences, 44, 94-103. Liu, X., Yan, Y., Li, F., \& Zhang, D. (2016). Fruit and vegetable consumption and the risk of depression: A meta-analysis. Nutrition, 32, 296-302.

Lucas, M., Mekary, R., Pan, A., Mirzaei, F., O’Reilly, E.J., Willett, W.C., . . . Ascherio, A. (2011). Relationship between clinical depression risk and physical activity and time spent watching television in older women: a 10-year prospective follow-up study. American Journal of Epidemiology, 174, 1017-1027.

Luppino, F.S., de Wit, L.M., Bouvy, P.F., Stijnen, T., Cuijpers, P., Penninx, B.W., \& Zitman, F.G. (2010). Overweight, obesity, and depression: a systematic review and meta-analysis of longitudinal studies. Archives of General Psychiatry, 67, 220-229.

Mammen, G., \& Faulkner, G. (2013). Physical activity and the prevention of depression: a systematic review of prospective studies. American Journal of Preventive Medicine, 45, 649-657.

Øvrum, A., Gustavsen, GW., \& Rickertsen, K. (2014). Age and socioeconomic inequalities in health: Examining the role of lifestyle choices. Advances in Life Course Research, 19, 1-13.

Piccinelli, M., \& Wilkinson, G. (2000). Gender differences in depression. Critical review. British Journal of Psychiatry, 177, 486-492.

Reyna,V.F., Nelson, W.L., Han, P.K., \& Pignone, M.P. (2015). Decision making and cancer. American Psychologist, 70, 105.

Saghafian, F., Malmir, H., Saneei, P., Milajerdi, A., Larijani, B., \& Esmaillzadeh, A. (2018). Fruit and vegetable consumption and risk of depression: accumulative evidence from an updated systematic review and meta-analysis of epidemiological studies. British Journal of Nutrition 119, 1087-1101.

Sarris, J., Kavanagh, D., \& Newton, R. (2008). Depression and exercise. Journal of Alternative and Complementary Medicine, 3, 48-50.

Sarris, J., O’Neil, A., Coulson, C.E., Schweitzer, I., \& Berk, M. (2014). Lifestyle medicine for depression. BMC Psychiatry, 14, 107. doi: 10.1186/1471-244X-14-107.

Schuit, J., van Loon, J., Tijhuis, M., \& Ocke, M. (2002) Clustering of lifestyle risk factors in a general adult population. Preventive Medicine, 35, 219-224.

Shojaee, M. (2014). Mental health components and locus of control. Final thesis for the master degree. Mount Saint Vincent University.

Short, S.E., \& Mollborn, S. (2015). Social Determinants and Health Behaviors: Conceptual Frames and Empirical Advances. Current Opinion in Psychology, 5, 78-84.

Sibille, E., \& French, B. (2013) Biological substrates underpinning diagnosis of major depression. The International Journal of Neuropsychopharmacology, 16, 1893-909. doi: $10.1017 / \mathrm{S} 1461145713000436$.

Slavin, J.L., \& Lloyd, B. (2012). Health benefits of fruits and vegetables. Advances in Nutrition, 3, 506-516.

Smithson, S., \& Pignone, M.P. (2017). Screening Adults for Depression in Primary Care. The Medical Clinics of North America, 101, 807-821. doi: 10.1016/j.mcna.2017.03.010.

Stringhini, S., Sabia, S., Shipley, M., Brunner, E., Nabi, H., Kivimaki, M., \& Manoux-Singh, A. (2010). Association of Socioeconomic Position With health Behaviors and Mortality. Journal of American Medical Association, 303, 1159-1166.

Swami, V., Vintila, M., Tudorel, O., Goian, C., \& Barron, D. (2018). Factor Structure and Psychometric Properties of a Romanian Translation of the Drive for Muscularity 
Scale. Body Image, 25, 48-55.

Teychenne, M., Ball, K., \& Salmon, J. (2008). Physical activity and likelihood of depression in adults: a review. Preventive Medicine, 46, 397-411.

Warburton, D.E., Nicol, C.W., \& Bredin, S.S. (2006) Health benefits of physical activity: the evidence. Canadian Medical Association Journal, 174, 801-809.

Weinberger, A.H., Pilver, C.E., Desai, R.A., Mazure, C.M., \& McKee, S.A. (2012). The relationship of dysthymia, minor depression, and gender to changes in smoking for current and former smokers: Longitudinal evaluation in the U.S. population. Drug and Alcohol Dependence, 127, 170-176.

World Health Organization. (2003). Fruit and vegetables promotion initiative - report of the meeting. Geneva: 25-27.

World Health Organization. (2009). Global health risks: mortality and burden of disease attributable to selected major risks. Geneva: World Health Organization.

World Health Organization. (2010). Global recommendations on physical activity for health.

Zhang, D., Giabbanelli, PJ., Arah, O.A., \& Zimmerman, FJ. (2014). Impact of Different Policies on Unhealthy Dietary Behaviors in an Urban Adult Population: An AgentBased Simulation Model. American Journal of Public Health, 104, 1217-1222. 